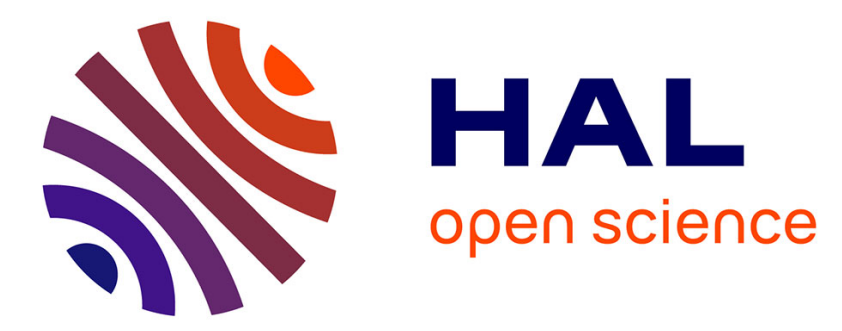

\title{
Zonal Detached Eddy Simulation (ZDES) using turbulent inflow and high order schemes: application to jet flows.
}

\author{
F. Gand, V. Brunet, G. Mancel
}

\section{- To cite this version:}

F. Gand, V. Brunet, G. Mancel. Zonal Detached Eddy Simulation (ZDES) using turbulent inflow and high order schemes: application to jet flows.. 5th Symposium on Hybrid RANS-LES Methods, Mar 2014, COLLEGE STATION, United States. hal-01057962

\section{HAL Id: hal-01057962 \\ https: / hal-onera.archives-ouvertes.fr/hal-01057962}

Submitted on 25 Aug 2014

HAL is a multi-disciplinary open access archive for the deposit and dissemination of scientific research documents, whether they are published or not. The documents may come from teaching and research institutions in France or abroad, or from public or private research centers.
L'archive ouverte pluridisciplinaire HAL, est destinée au dépôt et à la diffusion de documents scientifiques de niveau recherche, publiés ou non, émanant des établissements d'enseignement et de recherche français ou étrangers, des laboratoires publics ou privés. 


\title{
Zonal Detached Eddy Simulation (ZDES) using Tur- bulent Inflow and High Order Schemes: Application to Jet Flows.
}

\author{
F. Gand, V. Brunet and G. Mancel \\ Onera - The French Aerospace Lab, F-92190 Meudon, France
}

\begin{abstract}
This paper presents a numerical investigation of a round jet using a synthetic method to generate some free-stream turbulence into the jet core. Besides, this work also aims at evaluating the added advantage of the use of highorder numerical schemes - namely the AUSM+P spatial scheme with MUSCL extrapolation of the $3^{\text {rd }}$ and $5^{\text {th }}$ order - for this type of simulations. Academic test cases are presented to illustrate the main properties of the numerical methods used, then the Zonal Detached Eddy Simulation (ZDES) of a round jet is scrutinized with an emphasis on the influence of the injected synthetic turbulence.
\end{abstract}

\section{Introduction}

In the framework of sustainable aviation, it is now commonly acknowledged that jet flow simulations using eddy-resolving approaches could trigger major improvements for the prediction of airframe noise, acoustic sources locations, pylon unsteady loading, thermal fatigue, etc. While RANS/LES and LES methods have proven to be mature enough to reproduce quantitatively jet flows, these methods need to reproduce accurately the flight conditions to be of use in an industrial context.

However, the turbulence rate at the engine primary and secondary exhausts is usually neglected in simulations while this type of feature has a strong impact on the physics of the jet $[1,2]$. Apart from the turbulent content generation, an increase in simulations accuracy can also be achieved using high order numerics, or at least by reducing the amount of numerical dissipation in the simulations.

This paper presents numerical investigations of turbulent content generation for eddy-resolving simulations of simple test cases of core jet and wall turbulence. The potential added advantage of the use of high-order numerical schemes for this type of simulations is evaluated. The main findings are used to simulate a low speed round jet and the results are compared to an experimental database. 


\section{Numerical methods}

Flow solver The simulations presented in this paper have been performed with the els $\boldsymbol{A}$ software developed at Onera which solves the compressible NavierStokes equations on structured multiblock meshes.

The time integration is performed using an implicit LU-SSOR algorithm and a second-order accurate backward Gear scheme. The number of sub-iterations is adjusted to reach a convergence of one order of magnitude of the inner iteration residuals to achieve second-order time accuracy..

For the spatial integration, the diffusive fluxes are discretized using a secondorder-accurate centered scheme. The convective terms are treated with the hybrid centered/upwind second-order-accurate AUSM+P scheme [3] using MUSCL extrapolation of the third or fifth order. This version of the AUSM scheme involves a "wiggle" sensor to minimize numerical viscosity by applying some upwinding only in areas where the solution displays strong oscillations, while the scheme is actually centered everywhere else [3]. The AUSM $+\mathrm{P}$ scheme is well suited to the low-speed applications presented in this paper since its dissipation is proportional to the fluid velocity.

Physical modelling Several physical approaches are considered accordingly to the test-case investigated. In section 3, no turbulence model is used for the convection test case and Large Eddy Simulations (LES) are performed using the Mixed Scale Model (MSM) subgrid scale model for boundary layer simulations.

In section 4, hybrid RANS/LES simulations are performed using the Zonal Detached Eddy Simulation (ZDES) [4]. In the present study, modes 1 and 2 of the ZDES are used. The ZDES mode 1, introduced to treat separations triggered by the geometry, relies on a user-defined zonal decomposition of the computational domain in RANS and DES areas. The hybrid length scale $\widetilde{d}_{Z D E S}^{I}$ entering the Spalart-Allmaras (SA) model is equal to:

$$
\widetilde{d}_{Z D E S}^{I}=\left\{\begin{array}{l}
d_{\text {wall }} \text { in RANS areas } \\
\min \left(d_{\text {wall }}, C_{D E S} \Delta_{\omega}\right) \text { in DES areas }
\end{array}\right.
$$

where $d_{\text {wall }}$ is the wall distance; $C_{D E S}$ is the original DES97 constant and $\Delta \omega$ is the subgrid length scale based on the local vorticity direction $\Delta_{\omega}=\sqrt{\bar{S}_{\omega}}$, where $\bar{S}_{\omega}$ is the average cross section of the cell normal to the vorticity vector $\omega$. Furthermore, the near-wall functions of the SA model are removed in LES areas for mode 1: $f_{v 1}=1, f_{v 2}=0, f_{w}=0$.

The ZDES mode 2 [4] was developed to deal with separations over smooth surfaces. Therefore it relies on a protection function $f_{d}$ - similar to the one employed in DDES [5] - to shield the attached boundary layers. The hybrid length scale reads:

$$
\tilde{d}_{Z D E S}^{I I}=d_{\text {wall }}-f_{d} \max \left(0, d_{\text {wall }}-C_{D E S} \Delta_{Z D E S}^{I I}\right)
$$


Zonal Detached Eddy Simulation (ZDES) using Turbulent Inflow and High Order Schemes: Application to Jet Flows.

The mode 2 of ZDES provides a zonal definition of the subgrid length scale so that in attached regions $\Delta_{Z D E S}^{I I}=\Delta_{\max }\left(\Delta_{\max }\right.$ being the characteristic mesh length necessary to shield the attached boundary layers) but in detached areas, the subgrid length scales revolves to $\Delta_{Z D E S}^{I I}=\Delta_{\omega}$ or $\Delta_{v o l}$. The switch between the two subgrid length scales is done automatically using a threshold value $f_{d 0}=0.8$ for the $f_{d}$ function (see [4]).

Synthetic Eddy Method To generate realistic turbulent inflow content, the Synthetic Eddy Method (SEM) [6] is used. The SEM is based on the generation of velocity fluctuations carried by synthetic eddies which are superimposed to a target mean flow to obtain a synthetic unsteady turbulent field used to feed an inlet boundary condition. The formulation relies on the prescription by the user of a target Reynolds stress tensor. As suggested by Jarrin, in the present study an isotropic Reynolds stress tensor with $2 / 3 . k$ for the normal stresses is used as target (the turbulent kinetic energy profile is either extracted from a RANS boundary layer profile or computed from a target turbulent rate for jets cases).

The length scale of turbulence $\sigma$ has also to be prescribed. For wall turbulence, Jarrin has proposed to compute $\sigma$ according to RANS provided turbulent kinetic energy and dissipation rate profiles, or it can be explicitly given by the user.

The SEM formulation of Jarrin and its modifications presented above are implemented in a python module which is coupled with the els $\boldsymbol{A}$ software using the external coupling feature for boundary conditions.

\section{Preliminary verifications on academic cases}

\subsection{Turbulence convection}

Test case and simulations parameters The same test case of turbulence convection introduced in Ref. [1] is used in the present study. The computational domain is a square cylinder without any walls, each face being periodic, of dimensions in the streamwise (x), normal (y) and transverse (z) directions $5 \mathrm{LxLxL}(\mathrm{L}$ $=0.2 \mathrm{~m}$ ). The mesh is cartesian in the region of interest (up to $\mathrm{x}=0.8 \mathrm{~L}$ ) of dimension $(\mathrm{L} / 100)^{3}$. The cells are stretched in the streamwise direction from $\mathrm{x}=0.8$ $\mathrm{L}$ to create a sponge layer at the exit of the computational domain. In total, the grid contains $4.10^{6}$ cells. The inlet Mach number is set at $\mathrm{M}=0.2\left(\mathrm{U}_{0}=70 \mathrm{~m} / \mathrm{s}\right)$ with atmospheric pressure and temperature conditions.

The inlet turbulence level is set at $T u_{0}=(2 / 3 k) / U_{0}$, which is used within the SEM formulation to build an isotropic target Reynolds stress tensor as described in section 2. Two values of turbulence length scale have been tested: $\sigma=$ 
$\mathrm{L} / 20$ and $\sigma=\mathrm{L} / 5$ to assess the effect of the mesh resolution of the eddies generated.

For the present test case, no turbulence modelling is used. MUSCL extrapolation of the $3^{\text {rd }}$ and $5^{\text {th }}$ order is used along with the AUSM+P scheme. The time step is $10^{-5} \mathrm{~s}$, and the number of sub-iterations of the gear scheme has been set to 8 to ensure the decrease of one order of magnitude of the inner iteration residuals.

Results and discussion Flow visualizations are shown in Fig. 1. The turbulent structures generated by the SEM are convected through the computational domain, then are dissipated when entering the sponge layer are expected.

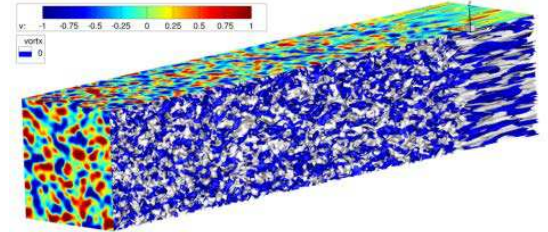

(a) $\sigma=L / 20$

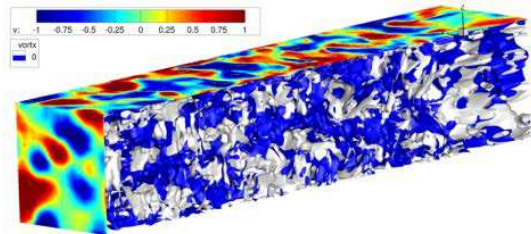

(b) $\sigma=L / 5$

Fig. 1 Flow visualizations for the turbulence convection test case. Isosurface of Q criterion colored by the streamwise vorticity and contours of transverse velocity

The streamwise evolution of the turbulence level is plotted in Fig. 2 (a). First, with the standard $3^{\text {rd }}$ order MUSCL extrapolation, it appears that the initial turbulence level is not sustained with only 5 grid points per eddy (case $\sigma=\mathrm{L} / 20$ ) whereas it is maintained with 20 grid points when $\sigma=\mathrm{L} / 5$. However, this situation seems to be improved when using a $5^{\text {th }}$ order reconstruction with $\sigma=\mathrm{L} / 20$. As a matter of fact, Fig. 2 (a) shows that there seems to be a significant additional amount of turbulent content resolved when the $5^{\text {th }}$ order MUSCL extrapolation is used, compared to the $3^{\text {rd }}$ one which is confirmed by the velocity spectra plotted in Fig. 2 (b). Even more, in the case $\sigma=\mathrm{L} / 20$, it seems that the solution recovers the $-5 / 3$ slope of the energy cascade, which could mean that the lack of grid points covering one eddy of length scale $\sigma=\mathrm{L} / 20$ is somewhat compensated by the increase of numerical accuracy.

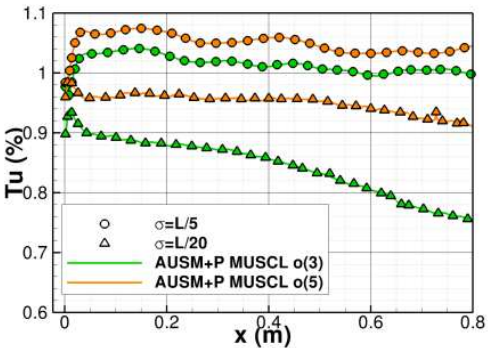

(a) Turbulence rate

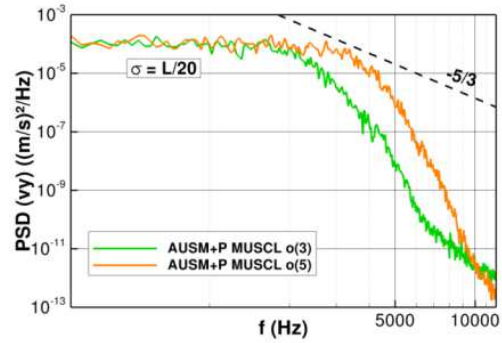

(b) Power Spectral Density

Fig. 2 Simple convection test case results: streamwise evolution of the turbulence rate (a) and PSD at $\mathrm{x}=0.6 \mathrm{~L}(\mathrm{~b})$

These results indicate that the SEM needs to be carefully set-up according to the inlet grid density (the reverse is also true, especially if inflow data is available from experiments) to achieve targeted inflow turbulence levels. In the next sec- 
Zonal Detached Eddy Simulation (ZDES) using Turbulent Inflow and High Order Schemes: Application to Jet Flows.

tion, the effect of the numerical accuracy is further to assess the application of the above conclusions to a more realistic case with a non-cartesian grid.

\subsection{Wall turbulence simulations}

In this section, the effect of the spatial scheme order in a Wall Resolved LES (WRLES) context is assessed.

Test case and simulations parameters A spatially developing turbulent boundary layer at a moderate Reynolds number $\left(\operatorname{Re}=4.10^{6} / \mathrm{m}\right)$ is investigated in the present section.

The inlet boundary layer thickness is $\delta_{0}=6.10^{-3} \mathrm{~m}$. The computational domain has been extended to $40 \delta_{0}$ in the streamwise direction $(\mathrm{x}), 4 \delta_{0}$ in the transverse direction (z) and $10 \delta_{0}$ in the wall normal direction (y). The cell sizes are $\Delta \mathrm{x}^{+}=50$, $\Delta \mathrm{z}^{+}=12$ and $\Delta \mathrm{y}^{+}=1$. The cell stretching ratio in the wall normal direction is less than $15 \%$. The mesh comprises $3.310^{6}$ cells. Reference DNS data for the station $\operatorname{Re}_{\theta}=1410$ are available in Ref. [7].

The simulations parameters are presented in section 2. The time step was set to $5.10^{-7} \mathrm{~s}$ so that the CFL number based on the maximum acoustic velocity is lower than 15 . The effect of the order of the MUSCL extrapolation $\left(3^{\text {rd }}\right.$ and $5^{\text {th }}$ order reconstruction) is assessed. Eight sub-iterations of the gear scheme were necessary to achieve a decrease of one order of magnitude of the inner iteration residuals.

Results and discussion A visualisation of the coherent structures resolved in a LES simulation performed in the present study is shown in Fig. 3. One can see that the typical boundary layer structures, including hairpin vortices, seem to be properly accounted for by the simulation.

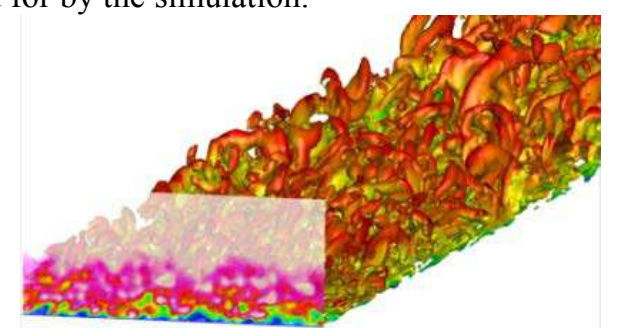

Fig. 3 Coherent structures highlighted by an isosurface of the Q criterion colored by the axial velocity and velocity contours generated with the SEM in the inlet plane

Fig. 4 (a) provides a more quantitative insight into the LES results and more specifically the effect of the increase of the accuracy of the spatial discretization. While the error on the friction coefficient at the reference location $x_{\operatorname{Re} \theta(x)=1410}$ is of $13 \%$ (compared to the RANS-SA solution) when using a standard $3^{\text {rd }}$ order MUSCL reconstruction - which is a classical result for this type of simulation us- 
ing this type of numerics - the error decreases to $7 \%$ with a $5^{\text {th }}$ order reconstruction.

In order to better understand the reasons of the increased accuracy of the simulations with high order MUSCL reconstruction, the Power Spectral Density (PSD) of the streamwise velocity at the location $x_{\operatorname{Re} \theta(x)=1410}$ at $\mathrm{y}+\approx 100$ is provided in Fig. 4 (b). The typical broadband shape of the boundary layer spectra is found, the energy cascade characterized by a slope of $-5 / 3$ of the velocity spectra is reproduced, although this phenomena occurs on a rather limited frequency bandwidth at this rather low Reynolds number. The focus is put on the frequency range $[10 ; 50]$ $\mathrm{kHz}$ to highlight some increase of the turbulent content resolved by the simulations when the $5^{\text {th }}$ order flux reconstruction is used.

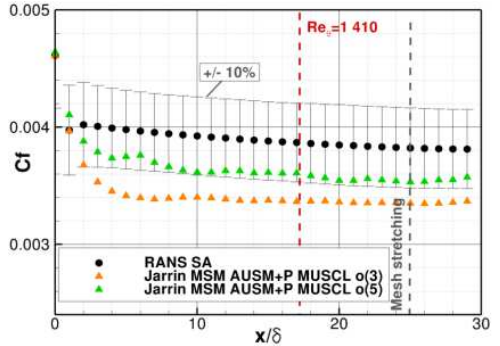

(a) Friction coefficient

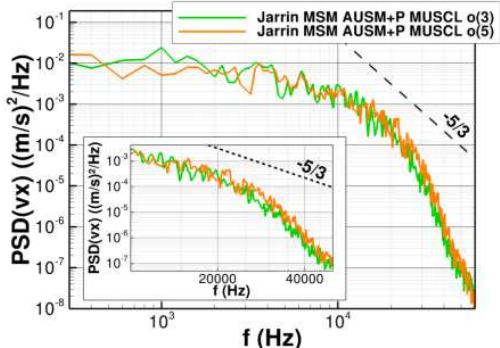

(b) $P S D$

Fig. 4 Boundary layer test case results: streamwise evolution of the friction coefficient (left) and PSD of the streamwise velocity velocity at $\operatorname{Re}_{\theta}=1410, y^{+} \approx 100$ (right)

As an intermediate conclusion, it can be noted that the use of high order MUSCL reconstruction with the AUSM+P scheme seems to be relevant in the framework of LES of wall turbulence. This is consistent with the results of section 3.1. However, the use of such schemes for more technical configurations (namely the round jet presented thereafter) leads to robustness issues which are still under examination.

\section{Application to a low speed round jet flow}

Axisymmetric jets have been widely investigated in the literature, both experimentally (e.g. [8]) and numerically (e.g. [2]). In particular, the influence of the initial level of perturbations in initially laminar boundary layers on the vortex pairing mechanisms and the associated noise generation is thoroughly documented. In the present study, initial turbulent boundary layers are considered and the focus is put on the effect of the core-jet perturbations, which is less commonly found in the literature.

Test case and simulations parameters The round jet investigated is similar to the one of Ref. [9]. The Reynolds number based on the nozzle exit velocity $\mathrm{U}_{0}=$ $20 \mathrm{~m} / \mathrm{s}$ and diameter $\mathrm{D}=0.15 \mathrm{~m}$ is equal to $\operatorname{Re}_{\mathrm{D}}=2.110^{5}$. 
Zonal Detached Eddy Simulation (ZDES) using Turbulent Inflow and High Order Schemes: Application to Jet Flows.

A sketch of the computational domain is presented in Fig. 5 (the actual grid is tri-dimensional, $\mathrm{x}$ is the axial coordinate). 40 grid points are used to discretize the nozzle boundary layer and the mesh contains $40.10^{6}$ cells in total. Three simulations were performed:

- Simulation 1: reference without any inflow turbulence, hence using mode 0 in the nozzle ("ZDES - no turb" in the following);

- Simulation 2: same as simulation 1, using the DDES approach [5] to assess the influence of the subgrid length scale used in the LES area;

- Simulation 3: simulation 1 with turbulence added at the nozzle inlet using SEM $\left(T u_{0}=2 \%\right.$ and $\sigma=0.02 \mathrm{~m}$, wild guess parameters) as shown in Fig. 5. In this case, mode 2 is retained inside the nozzle to allow both the transport of the injected turbulence and the RANS simulation of the attached boundary layer.

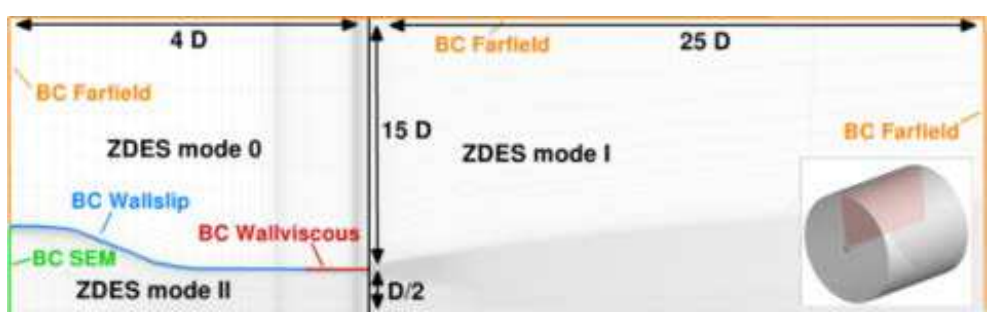

Fig. 5 Round jet test case. Slice in the 3D computational domain, ZDES settings and location of the boundary conditions.

Only the $3^{\text {rd }}$ order MUSCL reconstruction is assessed in the present paper since a simulation with the $5^{\text {th }}$ order has shown some robustness issues, the solving of which is underway. The time step is $2.10^{-6} \mathrm{~s}, 8$ sub-iterations are used for the gear scheme. The unsteady results are time averaged over $200 \mathrm{~ms}$ after a transient stage estimated of around $120 \mathrm{~ms}$.

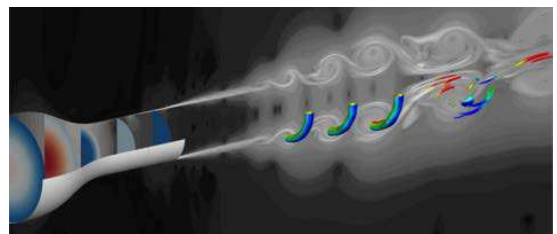

(a) DDES, no inlet turbulence

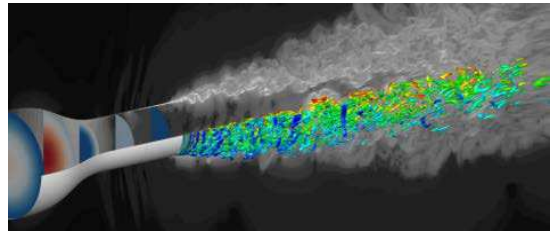

(b) ZDES, no inlet turbulence

Fig. 6 Flow visualizations. Isosurface of Q criterion, contours of density gradient (grayscale) and streamwise velocity inside the pipe (arbitrary scale)

Results and discussion Visualizations of simulations 1 and 2 are provided in Fig. 6. The quick growth of instabilities in the ZDES simulation is attributed to the use of $\Delta \omega$, thanks to which the eddy viscosity scales on the grid density normal to the main vorticity direction. In this case, the grid density actually allows the resolution of fine turbulent scales in the round mixing layer but the length scale used in DDES creates unjustified high values of eddy viscosity due to anisotropic cells downstream of the nozzle, hence the undesired damping of the instabilities. Simulation 3, not depicted here, exhibits little visual difference with simulation 1 . 
The nozzle exit conditions are shown in Fig. 7 (a). The experimental mean boundary layer profile is correctly reproduced by the simulations operating in RANS mode in this area and one can see that, in simulation 3, some of the core jet turbulence actually penetrates the RANS modelled boundary layer, and is somewhat amplified. It is important to remind that the addition of perturbations into the nozzle boundary layer is not the purpose of this study so it is not considered a drawback of the simulations to exhibit such low resolved turbulence levels in the attached boundary layer.

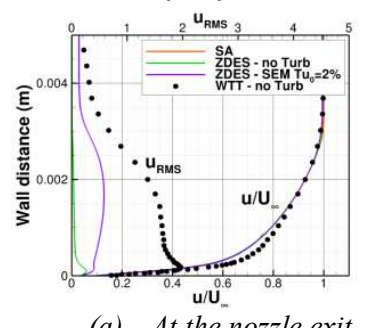

(a) At the nozzle exit

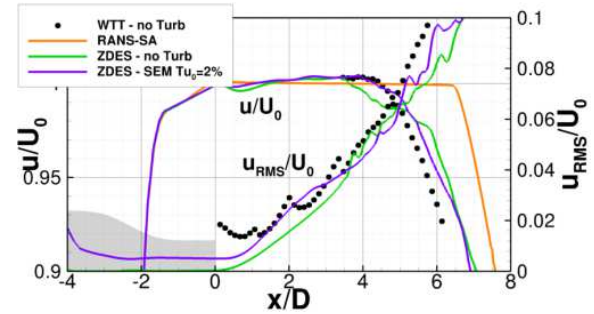

(b) Along the jet axis

Fig. 7 Axial mean velocity and RMS fluctuations (a) at the nozzle exit and (b) along the jet axis

The streamwise evolution of the velocity along the jet axis shown in Fig. 7 (b) indicates that the ZDES simulations actually succeed in predicting the potential core length measured in the experiments, whereas a RANS-SA simulation on the same mesh predicts a too long jet. However, it is difficult to assess the difference between the two ZDES simulations regarding the potential core length.

The injected fluctuations in simulation 3 undergo a strong decrease right downstream from the inlet plane, which is due to the fluid acceleration but is also attributed to a somewhat too coarse grid in the axial direction compared to the length scale of the eddies injected ( $\Delta \mathrm{x} \sim \sigma$ at the inlet). Therefore the turbulence level reached at the nozzle exit in simulation 2 is $0.5 \%$ only, which makes a small difference with simulation 1 but is actually consistent with the residual turbulence level measured experimentally.

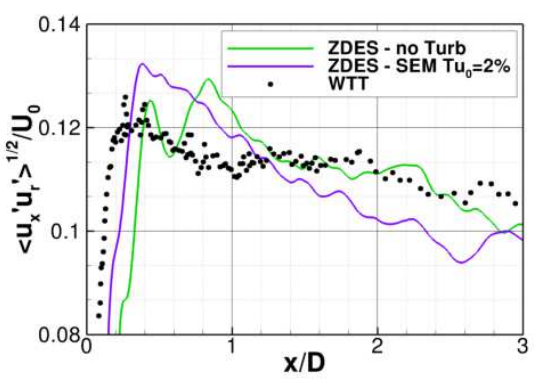

(a)
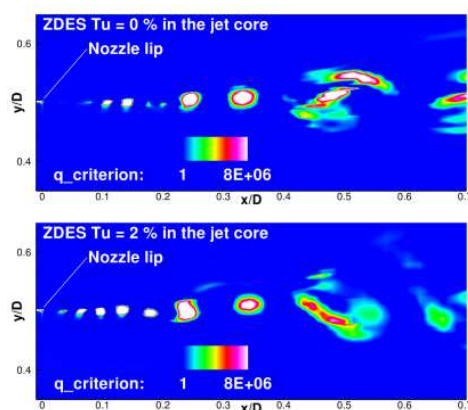

(b)

Fig. 8 Evolution of $\sqrt{\overline{u_{x}^{\prime} u_{r}^{\prime}}} / U_{0}$ in the shear layer (a) and Q criterion contours in the vicinity of the nozzle lip (b)

The very start of the formation of instabilities in the mixing layer is illustrated in Fig. 8. While the quick development of turbulence is confirmed for simulation 1, 
Zonal Detached Eddy Simulation (ZDES) using Turbulent Inflow and High Order Schemes: Application to Jet Flows.

the double peaked shaped evolution of $\sqrt{\overline{u_{x}^{\prime} u_{r}^{\prime}}} / U_{0}$ evidences the first stage of strong vortex pairings $[8]$ in this simulation which are not observed experimentally. Since no such shape is found in simulation 3 - which is therefore in better agreement with experimental data - it is advocated that the addition of turbulence into the core jet somewhat modifies the first steps of the mixing layer transition. This is all the more plausible since it appears from Fig. 7 (a) that the initial turbulent boundary layer incorporates some perturbations coming from the jet core. Fig. 8 (b) illustrates the even quicker development of the mixing layer in simulation 3 compared to simulation 1 .

The evolution of the frequency distribution of the turbulent energy along the jet axis and the mixing layer is plotted in Fig. 9. No differences are identified within the mixing layer. On the other hand, the flow clearly exhibits the signature of the inflow turbulence along the jet axis up to $\mathrm{x}=2 \mathrm{D}$. Increased low frequencies in simulation 2 are attributed to the eddies injected, but some spurious accumulation of energy at high frequencies is also observed. This issue is still under analysis but it could be a major limitation to the use of SEM methods in the framework of acoustic studies.

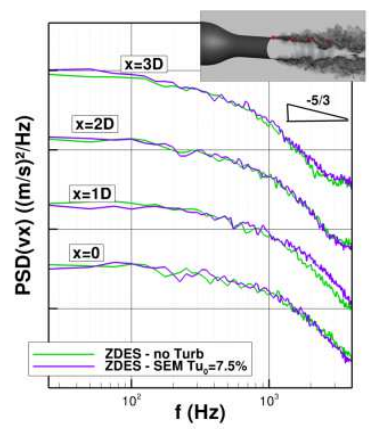

(a) In the mixing layer

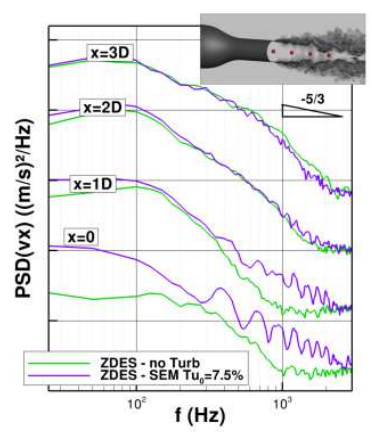

(b) Along the jet axis

Fig. 9 Power Spectral Density of the axial velocity fluctuations

\section{Summary, conclusions and future work}

Some preliminary work on turbulence injection and high order schemes in the context of RANS/LES simulations has been presented.

The use of the SEM allows a straightforward turbulence injection for jet flows, however its use imposes new constraints on the mesh in order to correctly advect the injected fluctuations.

The added advantage of using high order MUSCL reconstruction has been demonstrated on very basic test cases and remains to be confirmed on a more technical configuration for which robustness issues have been encountered.

The round jet simulation has highlighted the capability of the ZDES approach to simulate such flow without any delay in the formation of the instabili- 
ties. In particular, the use of a flow dependent subgrid length scale is advocated and shows that the removal of the eddy viscosity in the LES areas is not necessary to avoid delays in the formation of instabilities. It has been observed that the freestream turbulence generated in the ZDES simulation actually penetrates into the outer part of the nozzle boundary layer, which seems to influence the first stages of the shear layer development.

In the end, it is important to note that the possibility to add free stream turbulence for propulsive jet simulations brings into focus the need for detailed experimental data for these parameters to increase the experimental-numerical complementarity.

Acknowledgments The simulations presented in section 4 were performed using HPC resources from GENCI-TGCC (Grant 2014-t20142a7215).

\section{References}

[1] V. Brunet, "Random Flow Generation Technique for Civil Aircraft Jet Simulations with the ZDES Approach," in Progress in Hybrid RANS-LES Modelling, NNFM 117, 2012.

[2] C. Bogey, O. Marsden and C. Bailly, "Influence of Initial Turbulence Level on the Flow and Sound Fields of a Subsonic Jet at a Diameter-Based Reynolds Number of 10,", Journal of Fluid Mechanics, vol. 701, pp. 352-385, 2012.

[3] I. Mary and P. Sagaut, "Large Eddy Simulation of Flow around an Airfoil Near Stall," AIAA Journal, vol. 40 (6), pp. 1139-1145, 2002.

[4] S. Deck, "Recent Improvements of the Zonal Detached Eddy Simulation (ZDES) Formulation," Theoretical and Computational Fluid Dynamics, vol. 26, pp. 523-550, 2012.

[5] P. Spalart, S. Deck, M. Shur, K. Squires, M. Strelets and A. Travin, "A New Version of Detached-Eddy Simulation, Resistant to Ambiguous Grid Densities," Theoretical and Computational Fluid Dynamics, vol. 20, pp. 181-195, 2006.

[6] N. Jarrin, R. Prosser, J.-C. Uribe, S. Benhamadouche and D. Laurence, "Reconstruction of Turbulent Fluctuations for Hybrid RANS/LES Simulations using a Synthetic-Eddy Method," International Journal of Heat and Fluid Flow, vol. 30 (3), pp. 435-442, 2009.

[7] P. Spalart, "Direct Simulation of a Boundary Layer up to Rt=1410," Journal of Fluid Mechanics, vol. 187, pp. 61-98, 1988.

[8] K. Zaman and A. Hussain, "Vortex Pairing in a Circular Jet under Controlled Excitation. Part 1. General Jet Response," Journal of Fluids Mechanics, vol. 101, pp. 449-491, 1980.

[9] S. Davoust, L. Jacquin and B. Leclaire, "Dynamics of $m=0$ and $m=1$ Modes and of Streamwise Vortices in a Turbulent Axisymmetric Mixing Layer," Journal of Fluids Mechanics, vol. 709, pp. 408-444, 2012. 